\title{
Organocatalytic Enantioselective Synthesis of Tertiary $\alpha$-Hydroxy Phosphonates
}

\author{
Hyun Joo Lee, Ji Hyeon Kim, ${ }^{\dagger}$ and Dae Young Kim* \\ Department of Chemistry, Soonchunhyang University, Asan, Chungnam 336-745, Korea. "E-mail: dyoung@sch.ac.kr \\ Department of Chemical and Bio Engineering, Kyungwon University, Seongnam 461-701, Korea \\ Received December 25, 2010, Accepted January 12, 2011
}

Key Words : Enamine catalysis, Aldol reaction, $\alpha$-Hydroxy phosphonates, $\alpha$-Keto phosphonates

$\alpha$-Hydroxy alkylphosphonates have received attention both as substrates for the preparation of other $\alpha$-substituted phosphonates, ${ }^{1}$ and because of their inhibitory activity towards several important groups of enzymes, including farnesyl protein transferase, renin, HIV protease, and 5-enolpyruvylshikimate-3-phosphate synthase, and various classes of protein tyrosine kinases and phosphatases. ${ }^{2}$ Although certainly some are known, ${ }^{3}$ examples where the a carbon is fully substituted are less common and methods for the synthesis of such compounds may be more limited. The pharmaceutical potential of these compounds has stimulated the development of methodology for their preparation, particularly in enantiomerically enriched forms. ${ }^{1}$ Since the pioneering work of Zhao for the enantioselective synthesis of tertiary $\alpha$-hydroxy phosphonates via aldol reaction, ${ }^{4}$ several groups reported the catalytic enantioselective synthesis of tertiary $\alpha$-hydroxy phosphonates using taddol, prolinamide, and bispidine derivatives as catalysts. ${ }^{5}$

As part of a research program related to the development of synthetic methods for the enantioselective construction of stereogenic carbon centers, ${ }^{6}$ we recently reported chiral bifunctional organocatalyst to be a highly selective catalyst for the enantioselective addition of active methines. ${ }^{7} \mathrm{We}$ envisioned that the assembly of a structurally well-defined chiral 1,2-diamine and binaphthyl scaffold could constitute a new class of bifunctional organocatalyst. The rigid binaph-
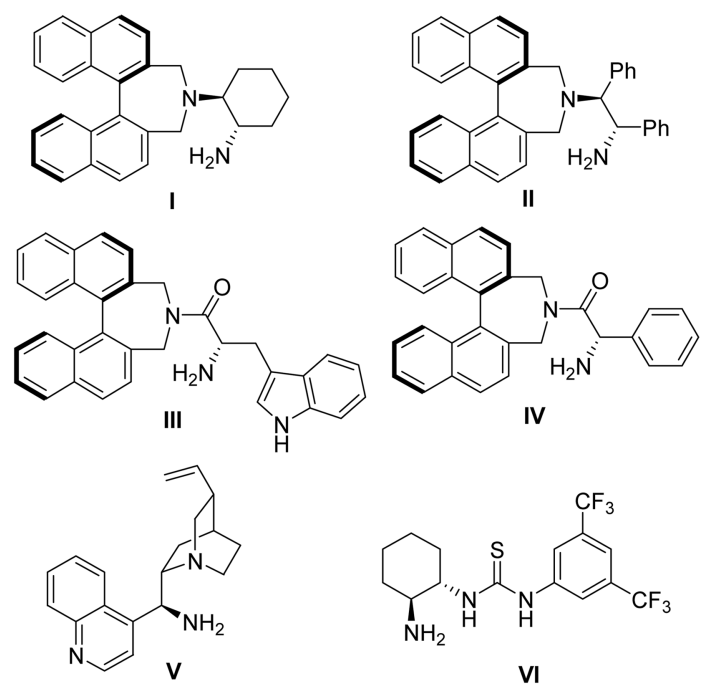

Figure 1. Structure of chiral primary amine catalysts. thyl structure can serve as an efficient stereocontrolling axial chiral element. Herein, we wish to describe the direct enantioselective aldol reaction of $\alpha$-keto phosphonates with acetone catalyzed by bifunctional organocatalysts bearing both central and axial chiral elements.

To determine suitable reaction conditions for the aldol reaction of $\alpha$-keto phosphonates, we initially investigated reaction system with $p$-toluoyl phosphonate (1a) and acetone (2) in the presence of $20 \mathrm{~mol} \%$ organocatalysts (Fig. 1) at room temperature. Some screening results are listed in Table 1. Chiral bifunctional organocatalyst I (20 mol \%) derived from $(1 S, 2 S)$-1,2-diaminocyclohexane promoted this reaction with $62 \%$ yield and $60 \%$ ee (Table 1 , entry 1). In the presence of benzoic acid as additive, enantioselectivity of this reaction was increased slightly (entry 2). Other primary amine catalysts such as binaphthyl group containing organocatalysts (II-IV), 9-amino-9-deoxy-epicinchonidine (V) and thiourea organocatalyst (VI) were also screened, but no superior result was obtained (entries 2-7). Based on the exploratory studies, we decided to select catalyst $\mathbf{I}$ for further optimization of reaction conditions. We examined our investigations by examining the reactivity and selectivity with organocatalyst $\mathbf{I}$ in the presence of different acids, such as formic acid, sulfonic acids, TFA, and substituted benzoic acids as additives (entries 2 and 8-13). Among the additives probed, the best results $(78 \%$ yield and $75 \%$ ee) were achieved when the reaction was conducted in 4-nitrobenzoic acid (entry 13). A survey of the reaction media indicated that many common solvents, such as toluene, DCM, THF, and diethyl ether (entries 13-16), were well tolerated in this aldol reaction with moderate to high enantioselectivities. THF offered slightly improved enantioselectivity (entry $15,79 \%$ ee). The present catalytic system tolerates catalyst loading down to $5 \mathrm{~mol} \%$ without compromising both yield and enantioselectivity (entry 17).

To examine the generality of this protocol, we studied the aldol reaction of acetone 2 to various $\alpha$-keto phosphonates 1a-f. As demonstrated in Table 2, organocatalyst I-catalyzed aldol reaction of acetone 2 with various $\alpha$-keto phosphonates 1 proved to be a general approach for the synthesis of chiral tertiary $\alpha$-hydroxy phosphonates 3 . Notably, good to high enantiomeric excess was obtained (up to $95 \%$ ee).

In conclusion, we have developed organocatalytic enantioselective synthesis of chiral tertiary $\alpha$-hydroxy phosphonates 3 via aldol reaction of acetone 2 and $\alpha$-keto phos- 
Table 1. Optimization of the reaction conditions

\begin{tabular}{|c|c|c|c|c|c|c|}
\hline & $1 a$ & & 2 & & $3 a$ & \\
\hline Entry & Cat. & Solvent & Additive & $\begin{array}{c}\text { Time } \\
\text { (h) }\end{array}$ & $\begin{array}{l}\text { Yield } \\
(\%)^{a}\end{array}$ & $\begin{array}{c}\text { ee } \\
(\%)^{b}\end{array}$ \\
\hline 1 & I & - & - & 9 & 62 & 60 \\
\hline 2 & I & - & $\mathrm{PhCO}_{2} \mathrm{H}$ & 6 & 60 & 71 \\
\hline 3 & II & - & $\mathrm{PhCO}_{2} \mathrm{H}$ & 6 & 63 & 29 \\
\hline 4 & III & - & $\mathrm{PhCO}_{2} \mathrm{H}$ & 7 & 41 & 51 \\
\hline 5 & IV & - & $\mathrm{PhCO}_{2} \mathrm{H}$ & 4 & 71 & 23 \\
\hline 6 & V & - & $\mathrm{PhCO}_{2} \mathrm{H}$ & 10 & 57 & 49 \\
\hline 7 & VI & - & $\mathrm{PhCO}_{2} \mathrm{H}$ & 15 & 53 & 15 \\
\hline 8 & I & - & $\mathrm{HCO}_{2} \mathrm{H}$ & 4 & 67 & 75 \\
\hline 9 & I & - & $(-) \mathrm{CSA}$ & 7 & 71 & 69 \\
\hline 10 & I & - & TfOH & 7 & 73 & 37 \\
\hline 11 & I & - & $p$-TsOH & 7 & 63 & 59 \\
\hline 12 & I & - & TFA & 9 & 76 & 75 \\
\hline 13 & I & - & $p-\mathrm{NO}_{2} \mathrm{C}_{6} \mathrm{H}_{4} \mathrm{CO}_{2} \mathrm{H}$ & 4 & 78 & 75 \\
\hline 14 & $\mathbf{I}$ & $\mathrm{CH}_{2} \mathrm{Cl}_{2}$ & $p-\mathrm{NO}_{2} \mathrm{C}_{6} \mathrm{H}_{4} \mathrm{CO}_{2} \mathrm{H}$ & 10 & 61 & 49 \\
\hline 15 & I & THF & $p-\mathrm{NO}_{2} \mathrm{C}_{6} \mathrm{H}_{4} \mathrm{CO}_{2} \mathrm{H}$ & 8 & 78 & 79 \\
\hline 16 & I & $\mathrm{Et}_{2} \mathrm{O}$ & $p-\mathrm{NO}_{2} \mathrm{C}_{6} \mathrm{H}_{4} \mathrm{CO}_{2} \mathrm{H}$ & 8 & 81 & 65 \\
\hline $17^{c}$ & I & THF & $p-\mathrm{NO}_{2} \mathrm{C}_{6} \mathrm{H}_{4} \mathrm{CO}_{2} \mathrm{H}$ & 9 & 80 & 79 \\
\hline
\end{tabular}

${ }^{a}$ Isolated yield. ${ }^{b}$ Enantioselectivity was determined by chiral HPLC analysis with Chiralpak AD-H. ${ }^{c} 5 \mathrm{~mol} \%$ catalyst loading.

Table 2. Enantioselective synthesis of $\alpha$-hydroxy phosphonates

\begin{tabular}{|c|c|c|c|c|}
\hline$\left(\mathrm{R}^{1} \mathrm{O}\right.$ & 2 & $\begin{array}{l}\mathrm{I}\left(5 \mathrm{~mol}^{\mathrm{I}}\right) \\
\mathrm{O}_{2} \mathrm{C}_{6} \mathrm{H}_{4} \mathrm{CO}_{2} \mathrm{H} \\
10 \mathrm{~mol} \%) \\
\mathrm{rHF}, \mathrm{rt}\end{array}$ & $\begin{array}{c}\left(\mathrm{R}^{1} \mathrm{O}\right)_{2} \mathrm{P}^{\mathrm{O}} \\
3^{\mathrm{R}} \\
\end{array}$ & \\
\hline Entry & $\mathbf{1}, \mathrm{R}^{1}, \mathrm{R}^{2}$ & Time (h) & Yield $(\%)^{a}$ & ee $(\%)^{b}$ \\
\hline 1 & $\mathrm{Et}, p-\mathrm{Me}-\mathrm{C}_{6} \mathrm{H}_{4}$ & 9 & $3 a, 80$ & 79 \\
\hline 2 & $\mathrm{Et}, p-\mathrm{F}-\mathrm{C}_{6} \mathrm{H}_{4}$ & 9 & $\mathbf{3 b}, 78$ & 83 \\
\hline 3 & $\mathrm{Et}, p-\mathrm{Br}-\mathrm{C}_{6} \mathrm{H}_{4}$ & 9 & 3c, 75 & 85 \\
\hline 4 & Et, Et & 15 & 3d, 71 & 87 \\
\hline 5 & $i-\mathrm{Pr}, p-\mathrm{Me}-\mathrm{C}_{6} \mathrm{H}_{4}$ & 6 & 3e, 78 & 95 \\
\hline 6 & $i-\mathrm{Pr}, \mathrm{Ph}$ & 4 & 3f, 77 & 85 \\
\hline
\end{tabular}

${ }^{a}$ Isolated yield. ${ }^{b}$ Enantiomeric excess was determined by HPLC analysis using chiral columns (Chiralpak AD-H for $\mathbf{3 a}, \mathbf{3 c}$, IA for $\mathbf{3 b}, \mathbf{3 e}$, and Chiralcel OJ for 3f) and GC analysis using CP-Chirasil-Dex CB for $\mathbf{3 d}$.

phonates 1. This reaction proceeds smoothly to afford the desired tertiary $\alpha$-hydroxy phosphonates in high yield and enantioselectivity. Further details and application of this asymmetric aldol reaction of keto phosphonates will be presented in due course.

Acknowledgments. Following are results of a study on the "Human Resource Development Center for Economic Region Leading Industry" Project, supported by the Ministry of Education, Science \& Technology (MEST) and the National Research Foundation of Korea (NRF).

\section{References and Notes}

1. For a review that includes synthesis of $\alpha$-hydroxy phosphonates, see: (a) Wiemer, D. F. Tetrahedron 1997, 53, 16609. (b) Kolodiazhnyi, O. I. Tetrahedron: Asymmetrty 2005, 16, 3295. (c) Merino, P.; Marques-Lopez, E.; Herrera, R. P. Adv. Synth. Catal. 2008, 350, 1195. 2. (a) Pompliano, D. L.; Rands, E.; Schaber, M. D.; Mosser, S. D.; Anthony, N. J.; Gibbs, J. B. Biochemistry 1992, 31, 3800. (b) Hohl, R. J.; Lewis, K. A.; Cermak, D. M.; Wiemer, D. F. Lipids 1998, 33, 39. (c) Patel, D. V.; Rielly-Gauvin, K.; Ryono, D. E. Tetrahedron Lett. 1990, 31, 5591. (d) Stowasser, B.; Budt, K. H.; Jian-Qi, L.; Peyman, A.; Ruppert, D. Tetrahedron Lett. 1992, 33, 6625. (e) Sikorski, J. A.; Miller, M. J.; Braccolino, D. S.; Cleary, D. G.; Corey, S. D.; Font, J. L.; Gruys, K. J.; Han, C. Y.; Lin, K. C.; Pansegrau, P. D.; Ream, J. E.; Schnur, D.; Shah, A.; Walker, M. C. Phosphorus Sulfur Silicon Relat. Elem. 1993, 76, 115.

3. (a) McEldoon, W. L.; Wiemer, D. F. Tetrahedron 1995, 51, 7131. (b) Hammerschmidt, F.; Schmidt, S. Eur. J. Org. Chem. 2000, 2239. (c) Kim, D. Y.; Wiemer, D. F. Tetrahedron Lett. 2003, 44, 2803. (d) Zhou, X.; Liu, Y.; Zhao, J.; Shang, D.; Liu, X.; Lin, L.; Feng, X. Adv. Synth. Catal. 2009, 351, 2567.

4. Samanta, S.; Zhao, C. G. J. Am. Chem. Soc. 2006, 128, 7442.

5. (a) Liu, J.; Yang, Z. G.; Wang, Z.; Wang, F.; Chen, X. H.; Liu, X. H.; Feng, X. M.; Su, Z. S.; Hu, C. W. J. Am. Chem. Soc. 2008, 130, 5654. (b) Huang, J. L.; Wang, J.; Chen, X. H.; Wen, Y. H.; Liu, X. H.; Feng, X. M. Adv. Synth. Catal. 2008, 350, 287. (c) Chen, X. H.; Wang, J.; Zhu, Y.; Shang, D. J.; Gao, B.; Liu, X. H.; Feng, X. M.; Su, Z. S.; Hu, C. W. Chem-Eur. J. 2008, 14, 10896. (d) Mandal, T.; Samanta, S.; Zhao, C. G. Org. Lett. 2007, 9, 943. (e) Gondi, V. B.; Hagihara, K.; Rawal, V. H. Angew. Chem., Int. Ed. 2009, 48, 776.

6. (a) Kim, D. Y.; Huh, S. C.; Kim, S. M. Tetrahedron Lett. 2001, 42, 6299. (b) Kim, D. Y.; Huh, S. C. Tetrahedron 2001, 57, 8933. (c) Kim, D. Y.; Park, E. J. Org. Lett. 2002, 4, 545. (d) Park, E. J.; Kim, M. H.; Kim, D. Y. J. Org. Chem. 2004, 69, 6897. (e) Kim, S. M.; Kim, H. R.; Kim, D. Y. Org. Lett. 2005, 7, 2309. (f) Kang, Y. K.; Kim, D. Y. Tetrahedron Lett. 2006, 47, 4565. (g) Kang, Y. K.; Cho, M. J.; Kim, S. M.; Kim, D. Y. Synlett 2007, 1135. (h) Lee, J. H.; Bang, H. T.; Kim, D. Y. Synlett 2008, 1821. (i) Kang, Y. K.; Kim, D. Y. Bull. Korean Chem. Soc. 2008, 29, 2093 (j) Kim, D. Y. Bull. Korean Chem. Soc. 2008, 29, 2036. (k) Kim, D. Y. Bull. Korean Chem. Soc. 2008, 29, 2036. (1) Mang, J. Y.; Whang, I. S.; Kwon, D. G.; Kim, D. Y. J. Korean Chem. Soc. 2008, 52, 724. (m) Mang, J. Y.; Kwon, D. G.; Kim, D. Y. J. Fluorine Chem. 2009, 130, 259. (n) Kang, S. H.; Kang, Y. K.; Kim, D. Y. Tetrahedron 2009, 65, 5676. (o) Mang, J. Y.; Kwon, D. G.; Kim, D. Y. Bull. Korean Chem. Soc. 2009, 30, 249. (p) Lee, N. R.; Kim, S. M.; Kim, D. Y. Bull. Korean Chem. Soc. 2009, 30, 829. (q) Kim, E. J.; Kang, Y. K.; Kim, D. Y. Bull. Korean Chem. Soc. 2009, 30, 1437. (r) Kang, S. H.; Kim, D. Y. Bull. Korean Chem. Soc. 2009, 30, 1439. (s) Kwon, B. K.; Kim, D. Y. Bull. Korean Chem. Soc. 2009, 30, 1441. (t) Kang, Y. K.; Kim, D. Y. Curr. Org. Chem. 2010, 14, 917. (u) Kang, S. H.; Kim, D. Y. Adv. Synth. Catal. 2010, 352, 2783. (v) Kang, Y. K.; Kim, S. M.; Kim, D. Y. J. Am. Chem. Soc. 2010, 132, 11847.

7. (a) Kim, S. M.; Lee, J. H.; Kim, D. Y. Synlett 2008, 2659. (b) Jung, S. H.; Kim, D. Y. Tetrahedron Lett. 2008, 49, 5527. (c) Kang, Y. K.; Kim, D. Y. J. Org. Chem. 2009, 74, 5734. (d) Lee, J. H.; Kim, D. Y. Adv. Synth. Catal. 2009, 351, 1779. (f) Kwon, B. K.; Kim, S. M.; Kim, D. Y. J. Fluorine Chem. 2009, 130, 759. (g) Oh, Y.; Kim, S. M.; Kim, D. Y. Tetrahedron Lett. 2009, 50, 4674. (h) Moon, H. W.; Cho, M. J.; Kim, D. Y. Tetrahedron Lett. 2009, 50, 4896. (i) Kang, Y. K.; Kim, D. Y. J. Org. Chem. 2009, 74, 5734. (j) Lee, J. H.; Kim, D. Y. Adv. Synth. Catal. 2009, 351, 1779. (k) Lee, J. H.; Kim, D. Y. Synthesis 2010, 1860. (1) Moon, H. W.; Kim, D. Y. Tetrahedron Lett. 2010, 51, 2906.

8. General procedure for asymmetric synthesis of $\alpha$-hydroxy alkylphosphonates 3: To a stirred solution of $\alpha$-keto phosphonate (1, $0.3 \mathrm{mmol}), 4$-nitrobenzoic acid $(5.0 \mathrm{mg}, 0.03 \mathrm{mmol})$, and catalyst I $(5.8 \mathrm{mg}, 0.015 \mathrm{mmol})$ in THF $(0.7 \mathrm{~mL})$ was added acetone $(0.66$ $\mathrm{mL}, 9 \mathrm{mmol})$. Reaction mixture was stirred for $4-15 \mathrm{~h}$ at room temperature, concentrated, and purified by flash column chromatography (EtOAc) to afford the aldol product 3. 